\title{
Atitudes de Estudantes de Medicina em relação ao Suicídio
}

\author{
Attitudes of Medical Students toward \\ Suicide
}

\author{
Claudia Antunes Magalhães ${ }^{I}$ \\ Diego Murilo Miranda Neves ${ }^{I}$ \\ Leandro Martins Drumond Moreira Brito ${ }^{I}$ \\ Bárbara Bravo Cruz Leite ${ }^{I}$ \\ Mônica Maria de Faria Pimenta ${ }^{I}$ \\ Carlos Eduardo Leal Vidal ${ }^{I I}$
}

\section{PALAVRAS-CHAVE \\ - Suicídio; \\ - Estudantes; \\ - Atitudes; \\ - Medicina; \\ - Educação Médica.}

\section{KEYWORDS}

- Suicide;

- Students;

- Attitudes;

- Medicine;

- Medical Education.

\begin{abstract}
RESUMO
Introdução: Profissionais e estudantes da área da saúde atendem indivíduos deprimidos em situação de crise e que tentam suicídio, e as atitudes em relação a esses pacientes podem ser determinantes na evolução dos casos. Objetivo: Verificar as atitudes de estudantes frente ao paciente suicida. Método:Trata-se de estudo com delineamento transversal realizado com graduandos da Faculdade de Medicina de Barbacena(MG) em 2013. Os estudantes foram divididos em dois grupos: pré-clinico e pós-clínico. O instrumento utilizado neste estudo foi o Questionário de Atitudes em Relação ao Comportamento Suicida. Resultados: A comparação dos escores médios obtidos em cada grupo evidenciou diferença significativa, com maior escore apresentado pelo grupo pós-clínico ( $p=0,028)$. Esse achado indica que estudantes de períodos mais avançados exibem atitudes mais positivas em relação ao suicídio que alunos iniciantes no curso de Medicina. Conclusão: Observou-se que um contato maior com o paciente suicida e maior conhecimento teórico sobre o suicídio e seus fatores determinantes podem ser capazes de modificar de forma favorável as atitudes dos estudantes.
\end{abstract}

Introduction: Both health professionals and students care forpatientsin depression, crisis, orare suicidal, and their attitudes toward such individuals can be a key factor in the outcome of cases. Objective:To verify students' attitudes towards suicidal patients. Method: A cross-sectional study was developed in 2013 with students from the Barbacena University Medical School, in the state of Minas Gerais. The medical students were evaluated and divided into two groups: pre-clinical and post-clinical. The instrument used in this study was the Questionnaire on Attitudes towards Suicidal Behavior. Results:Acomparison of the mean scores obtained in each group revealed a significant difference, with the higher score found among the post-clinical group ( $p=0.028$ ). This finding indicates that students in the later periods of their course exhibit more positive attitudes towards suicide when compared to beginner medical students. Conclusion:It was noted that more contact with suicidal patients and greater theoretical knowledge about suicide and its determinants helped improve the students' attitudes towards this issue. 


\section{INTRODUÇÃO}

O suicídio é um fenômeno humano complexo e universal e, juntamente com as tentativas, representa um grande problema de saúde pública. Segundo a Organização Mundial da Saúde $(\mathrm{OMS})^{1}$, as mortes por suicídio aumentaram $60 \%$ nas últimas cinco décadas. A taxa mundial de suicídio situa-se em torno de 16 por 100 mil habitantes e estima-se que mais de um milhão e meio de pessoas cometam suicídio em $2020^{2}$.

Em termos de anos de vida perdidos por incapacidade (Dalys - disability-adjusted life years), a OMS ${ }^{1}$ atribuiu ao suicídio quase $2 \%$ das perdas por doença em todo o mundo em 1998. Este impacto foi semelhante ao causado pelas guerras e homicídios, e corresponde ao dobro do causado pelo diabetes ${ }^{3}$.

Pelo menos $90 \%$ dos que cometem suicídio apresentam um transtorno psiquiátrico, predominantemente depressão, e mais de dois terços não estavam em tratamento quando morreram. A prevenção do suicídio é uma função crítica dos médicos, e o reconhecimento da depressão é o primeiro passo para preveni-lo, especialmente quando existe comorbidade com abuso de substâncias².

A maioria dos pacientes que comete suicídio é atendida por um médico da Atenção Básica antes de ser avaliada por psiquiatra. Entre os pacientes que cometeram suicídio, de $45 \%$ a $66 \%$ tiveram contato com um clínico durante o mês em que morreram, e de $10 \%$ a $40 \%$ procuraram seu médico uma semana antes de se suicidar ${ }^{1}$.

Ideação suicida está presente em até $10 \%$ dos pacientes atendidos em cuidados primários e essa taxa pode chegar a $60 \%$ naqueles portadores de depressão ${ }^{4}$. Pesquisas mostram que, embora os pacientes suicidas não expressem voluntariamente seus pensamentos suicidas quando na presença do médico, eles admitem esses pensamentos se interrogados diretamente sobre suicídio ${ }^{4,5}$, e que intervenções apropriadas podem aprimorar o diagnóstico de depressão e reduzir a incidência de suicídio ${ }^{6,7}$. Assim, os médicos têm um papel crítico em detectar pacientes deprimidos que exibem maior risco de suicídio, para os quais existem estratégias terapêuticas efetivas ${ }^{5}$.

Esses aspectos mostram que o conhecimento sobre depressão e suicídio, englobando desde fatores causais até estratégias terapêuticas, é um componente relevante na formação dos profissionais de saúde, especialmente médicos, enfermeiros e psicólogos. Considerando a importância do tema, este trabalho teve por objetivo investigar e comparar as atitudes de graduandos do curso de Medicina frente a uma situação relevante da prática médica - o suicídio.

\section{MÉTODO}

\section{Tipo de estudo}

Trata-se de estudo com delineamento transversal realizado com graduandos da Faculdade de Medicina de Barbacena,Minas Gerais. A faculdade tem quatro décadas de existência e conta com aproximadamente 700 estudantes matriculados. A cidade é referência regional para 15 municípios e compreende uma população aproximada de 230 mil habitantes. Foram avaliados alunos iniciantes e concluintes, divididos em dois grupos: o pré-clínico, representado por estudantes do primeiro ao sétimo período, e o pós-clínico, compreendendo aqueles do oitavo ao $12^{\circ}$ período.

\section{Instrumento}

O instrumento utilizado foi o Questionário de Atitudes em Relação ao Comportamento Suicida (SBAQ) ${ }^{8}$. Ele é formado por 25 itens que constituem uma escala visual analógica, ou seja, uma linha contínua de $100 \mathrm{~mm}$ cujas respostas vão desde "discordo totalmente" numa extremidade a "concordo totalmente" na outra. O entrevistado é convidado a indicar um ponto em cada linha, o que melhor reflete suas opiniões, sentimentos e reações.O estudo de validaçãomostrou que o referido instrumento é de fácil manuseio e bem simples para avaliar a atitude em relação aos suicidas. O questionário inclui também informações sobre religião, frequência a cultos religiosos, experiência no atendimento ao paciente suicida e contato familiar com suicida.

\section{Análise estatística}

Foram construídas tabelas para distribuição de frequências, médias, medianas e desvio-padrão (DP) para cada variável. Depois da determinação dos escores da escala para cada participante, os subgrupos da amostra foram comparados conforme as diferentes categorias da escala. Para comparação dos dois grupos de estudantes quanto aos resultados da escala e quanto às variáveis independentes, foram utilizados o teste $t$ de Student e o teste do Qui-Quadrado, conforme o caso. Diferenças foram consideradas significantes com $p<0,05$. Os dados foram lançados em planilhas no softwareExcel e processados pelo programa Stata 9.2.

\section{Considerações éticas}

O projeto de pesquisa foi aprovado pelo Comitê de Ética em Pesquisa da Universidade Presidente Antônio Carlos em 25/10/2012, sob o número 157.236. Todos os alunos assinaram o Termo de Consentimento Livre e Esclarecido. 


\section{RESULTADOS}

A amostra foi constituída por 349 estudantes cursando do primeiro ao $12^{\text {o }}$ período da Faculdade de Medicina de Barbacena, com idade média de 23,2ะ3,5 anos, variando de 17 a 44 anos. $\mathrm{O}$ sexo feminino compreendeu 226 indivíduos (64,8\%), e o sexo masculino, 123 (35,2\%).O grupo pré-clínico $(n=249)$ foi composto por 161 mulheres $(65,0 \%)$ e 88 homens (35,0\%); o pós-clínico $(n=100)$ por 65 mulheres $(65,0 \%)$ e 35 homens $(35,0 \%)$.

Em relação à religiosidade 192 (77,1\%) se declararam católicos no grupo pré-clínico, seguidos por 72 (72,0\%) no pós-clínico. Os demais foram classificados como sem religião e outras religiões. Nas respostas às perguntas sobre atendimento de pessoas com ideação suicida ou com tentativa de suicídio, percebeu-se diferença estatisticamente significante entre os dois grupos. Com relação ao questionamento sobre suicídio em familiar, não houve diferença entre os grupos. Os dados relativos às características da amostra, discriminados por grupo, são apresentados na Tabela 1.

\begin{tabular}{|c|c|c|c|c|c|c|}
\hline Caracter & $\begin{array}{r}\text { ticas de } \\
\text { Bar }\end{array}$ & $\begin{array}{r}\mathrm{T} \\
\operatorname{amos} \\
\text { acen }\end{array}$ & $\begin{array}{l}\text { ELA 1: } \\
\text { a discri } \\
2013-C\end{array}$ & $\begin{array}{l}\text { hinad } \\
\text { upos }\end{array}$ & por $\mathrm{g}$ & upo, \\
\hline Variáveis & $\begin{array}{l}\text { Pré- } \\
\text { clínico }\end{array}$ & & $\begin{array}{l}\text { Pós- } \\
\text { clínico }\end{array}$ & & Total & \\
\hline & n & $\%$ & $\mathbf{N}$ & $\%$ & $\mathbf{n}$ & Valor $p$ \\
\hline Sexo & & & & & & \\
\hline Feminino & 161 & 65 & 65 & 65 & 226 & 1,000 \\
\hline Masculino & 88 & 35 & 35 & 35 & 123 & \\
\hline Total & 249 & 100 & 100 & 100 & 349 & \\
\hline Religiosidade & & & & & & \\
\hline Ateu & 8 & 3 & 1 & 1 & 9 & 0,179 \\
\hline Religião & 227 & 91 & 94 & 97 & 321 & \\
\hline Sem religião & 14 & 6 & 2 & 2 & 16 & \\
\hline Total & 249 & 100 & 97 & 100 & 346 & \\
\hline $\begin{array}{l}\text { Atendimento } \\
\text { a suicida }\end{array}$ & & & & & & \\
\hline Não & 212 & 85 & 33 & 33 & 245 & 0,000 \\
\hline Sim & 36 & 15 & 66 & 67 & 102 & \\
\hline Total & 248 & 100 & 99 & 100 & 347 & \\
\hline $\begin{array}{l}\text { Atendimento } \\
\text { a suicida } \\
\text { internado }\end{array}$ & & & & & & \\
\hline Não & 210 & 85 & 33 & 33 & 243 & 0,000 \\
\hline Sim & 38 & 15 & 66 & 67 & 104 & \\
\hline Total & 248 & 100 & 99 & 100 & 347 & \\
\hline $\begin{array}{l}\text { Convívio com } \\
\text { suicida }\end{array}$ & & & & & & \\
\hline Não & 196 & 79 & 76 & 77 & 272 & 0,774 \\
\hline Sim & 53 & 21 & 23 & 23 & 76 & \\
\hline Total & 249 & 100 & 99 & 100 & 348 & \\
\hline
\end{tabular}

A comparação dos escores médios obtidos em cada grupo evidenciou diferença significativa, com maior escore sendo apresentado pelo grupo pós-clínico $(56,2 \pm 7,7)$ contra $54,2 \pm 7,5$ do grupo pré-clínico ( $\mathrm{p}=0,028)$.

De acordo com os autores da escala SBAQ, em seu processo de construção três fatores interpretáveis foram extraídos e denominados "sentimentos em relação ao paciente", "capacidade para lidar com situações que envolvem o comportamento suicida" e concepções sobre o "direito ao suicídio".

Nos fatores "sentimentos" e "capacidade", não se observaram diferenças significantes entre as respostas dos alunos dos grupos pré- e pós-clínico. Com relação aos "sentimentos", as atitudes foram consideradas positivas nos dois grupos. E quanto ao fator "capacidade", verificou-se dificuldade dos estudantes para lidar com situações que envolvam comportamento suicida.

No fator "direito ao suicídio", as pontuações mais altas foram consideradas mais favoráveis. Neste item houve diferença estatisticamente significante entre a média dos grupos pré-clínico e pós-clínico $(66,2 \pm 14,7$ e $70,5 \pm 13,7$, respectivamente; $\mathrm{p}=0,013$ ).

Algumas questões não foram relacionadas em nenhum dos agrupamentos citados e foram analisadas separadamente. Os resultados discriminados por grupos com as respectivas médias estão apresentados na Tabela 2 .

A análise das variáveis independentes considerando-se os grupos pré- e pós-clínico não evidenciou diferença entre os grupos com relação a sexo, religiosidade e convívio familiar com paciente suicida. Nas respostas às perguntas sobre atendimento ao suicida, percebeu-se diferença estatisticamente significante entre os dois grupos, com os alunos dos períodos mais avançados tendo mais contato com tais pacientes.

\section{DISCUSSÃO}

No presente estudo foram apresentados dados relacionados a conhecimento, atitudes e crenças de estudantes da Faculdade de Medicina de Barbacena em relação ao suicídio. Os resultados obtidos, embora não possam ser generalizados para outras instituições de ensino médico, fornecem subsídios que podem favorecer discussões sobre a formação médica em relação ao atendimento de pacientes que exibem comportamento suicida.

A morte, principalmente por suicídio, é um dos temas que oferece maiores dificuldades emocionais a todos os profissionais da área da saúde, o que parece mais evidente em estudantes de Medicina, quando comparados a médicos ou a estudantes de Enfermagem. Se não forem devidamente abordadas, essas dificuldades podem levar a problemas no relacionamento 


\section{TABELA 2:}

Escores médios de acordo com grupos pré- e pós-clínico, Barbacena, 2013

Resultado final por grupos
Q6 Quem fica ameaçando geralmente não se mata
Q9 No fundo, prefiro não me envolver muito com pacientes que tentaram o suicídio
Q13 Tenho receio de perguntar sobre ideias de suicídio e acabar induzindo o paciente a isso
Q17 No fundo, às vezes dá até raiva, porque tanta gente querendo viver... e aquele paciente
Q19 A gente se sente impotente diante de uma pessoa que quer se matar
Q21 No caso de pacientes que estejam sofrendo muito devido a uma doença física, acho
Q23 Quem queitável a ideia de suicídio matar mesmo não fica “tentando" se matar
Q23

\section{Pré-clínico \\ Média e DP}

$54,21(7,53)$

0,028

Q5 Me sinto capaz de ajudar uma pessoa que tentou se matar

$\begin{array}{lll}56,2(30,1) & 60,6(29,2) & 0,206 \\ 72,3(28,0) & 71,4(27,7) & 0,788 \\ 68,2(28,2) & 68,3(30,7) & 0,980 \\ 57,9(35,9) & 67,9(31,1) & 0,015 \\ 54,7(30,7) & 45,3(27,7) & 0,008 \\ 76,5(28,7) & 71,3(31,2) & 0,142 \\ 50,8(34,6) & 55,2(34,2) & 0,282 \\ 62,3(15,9) & \mathbf{6 2 , 8 ( 1 5 , 1 )} & \mathbf{0 , 7 9 2} \\ & & \end{array}$

Q11 Me sinto capaz de perceber quando um paciente tem risco de se matar

$\begin{array}{lll}47,9(29,6) & 45,1(27,8) & 0,406 \\ 35,2(24,4) & 36,1(25,0) & 0,756 \\ 15,6(20,1) & 21,2(19,5) & 0,018 \\ 39,6(30,0) & 30,2(26,2) & 0,006 \\ 34,6(16,2) & 33,2(15,6) & 0,462\end{array}$

Q14 Tenho preparo profissional para lidar com pacientes com risco de suicídio

$69,9(31,2)$

$71,35(29,3)$

0,703

Q7 Apesar de tudo, penso que, se uma pessoa deseja se matar, ela tem esse direito

Q8

Diante de um suicídio penso: se alguém tivesse conversado, a pessoa teria encontrado outro caminho

$60,1(27,3)$

$65,5(27,5)$

0,092

Q10 A vida é um dom de Deus, e só Ele pode tirar

$51,5(37,7)$

$64,9(33,8)$

0,002

Q20 Quem tem Deus no coração não vai tentar se matar

$73,8(32,1)$

$70,8(29,4)$

0,410

Q22 Quando uma pessoa fala em pôr fim à vida, tento tirar aquilo da cabeça dela

$75,8(27,7)$

$79,8(21,6)$

0,745

\section{Resultado final}

$66,2(14,7)$

$70,4(13,7)$

0,013

\section{Outras}

Q1 É difícil diferenciar se os pacientes se apresentam simplesmente infelizes ou se têm uma depressão que necessita de tratamento

Q2 Ficar deprimido é a maneira como pessoas mais frágeis lidam com dificuldades da vida
$51,7(28,9)$

$66,2(31,9)$

$59,4(30,0)$

$83,4(23,9)$

$29,7(30,2)$

$35,4(32,8)$

$51,3(26,9)$

$57,5(31,2)$

$14,9(27,8)$
$61,5(27,6)$

0,003

$66,8(30,4)$

0,861

$60,7(27,6)$

0,710

$78,1(25,8)$

0,068

$33,3(32,3)$

0,337

$34,4(31,5)$

0,801

Se eu sugerir uma interconsulta psiquiátrica para um paciente que falou em se matar, penso que isso será bem aceito pelo médico

Q24 Um paciente internado dificilmente se mata sem que tenha um forte motivo para isso

Q25 Eu já passei por situações que me fizeram pensar em cometer suicídio
$63,1(25,3) \quad 0,000$

$58,4(32,2)$

0,792

$23,4(29,7)$ 
com o paciente ou com seus familiares, interferir na atuação profissional e levar a reações contratransferenciais negativas ${ }^{9}$.

De forma global, a comparação das atitudes dos estudantes, tendo por base os escores médios obtidos, evidenciou a presença de mais atitudes positivas no grupo pós-clínico, em especial no resultado final do agrupamento "direito ao suicídio". Esse dado evidencia melhor compreensão do ato suicida como expressão de um transtorno psiquiátrico que necessita de abordagem e intervenção.

No agrupamento "sentimentos",não se observou diferença no resultado final entre os grupos avaliados. No entanto,consideradas separadamente, as questões 17 e 19 mostraram resultados desiguais. Na questão 17- “No fundo, às vezes dá até raiva, porque tanta gente querendo viver... e aquele paciente querendo morrer"-, o grupo pós-clínico demonstra menor preconceito e maior conhecimento teórico da condição psiquiátrica. Quanto à questão 19- "A gente se sente impotente diante de uma pessoa que quer se matar"-, o grupo pós-clínico revela maior insegurança e falta de confiança para atuar frente ao paciente suicida. Isso pode ser devidoa uma melhor visão da vivência profissional médica ao longo da graduação e ao medo de lidar com tal situação a curto prazo. $\mathrm{O}$ mesmo raciocínio se aplica à questão 16 - "Sinto-me inseguro(a) para cuidar de pacientes com risco de suicídio" - do grupamento "capacidade".

Considerando o fator "capacidade para lidar com situações que envolvem o comportamento suicida", observou-se que o resultado final foi desfavorável nos dois grupos de estudantes. Tal fato pode ser decorrente da falta de capacitação e conhecimento dos alunos, indicando que o tema deveria ser abordado de forma mais abrangente na graduação. Neste agrupamento, apenas as questões 14 e 16 (discutidas acima) apresentaram significância estatística. Na questão 14 - “Tenho preparo profissional para lidar com pacientes com risco de suicídio" -, o pós-clínico mostrou que os estudantes se consideram mais capazes de lidar com o paciente suicida.

Troncon et al. ${ }^{9}$ avaliaram as atitudes de estudantes de Medicina em relação a seis aspectos relevantes da prática médica, e, nos itens relativos à doença mental e à morte, a maioria dos alunos apresentou escores indicativos de atitudes negativas ou indefinidas. A incorporação de atitudes positivas frente a diferentes aspectos envolvidos na atenção à saúde constitui objetivo consensual da formação integral do graduando em Medicina.

Outro estudo ${ }^{10}$, que envolveu alunos do segundo e sexto ano de uma faculdade de Medicina sobre atitudes frente a diferentes aspectos da prática médica, evidenciou que os alunos do segundo ano apresentaram maior percentual de atitudes negativas em situações relacionadas à morte quando comparados aos alunos do último período. Este achado, segundo os autores, parece sugerir que, no decorrer do curso, o aluno teria experiências favorecedoras de reflexão e um posicionamento mais amadurecido diante das situações envolvidas com a morte. No entanto, o baixo escore obtido nos dois grupos de estudantes no referido estudo indica a complexidade que o tema morte assume para os alunos.

Em qualquer período de cursos de Medicina, os estudantes exibem forte crença de que pacientes com ideação suicida podem ser ajudados, mas as atitudes dos alunos são influenciadas pelo conhecimento adquirido sobre transtornos mentais e aspectos biológicos do comportamento durante o aprendizado. Assim, entre os estudantes que se encontram no final do curso observa-se uma visão mais médica em relação ao suicídio. E, de acordo com alguns estudos, as atitudes frente ao suicídio entre estudantes de Medicina influenciam o comportamento futuro que terão com esses pacientes ${ }^{8}$.

$\mathrm{Na}$ avaliação da competência para o atendimento ao paciente que tentou suicídio, esperavam-se atitudes mais ativas e colaborativas nos estudantes dos períodos pós-clínicos do que entre os estudantes do grupo pré-clínico. Entretanto, a avaliação das médias demonstrou uma tendência de atitude aproximada entre os dois grupos, reforçando a necessidade de capacitação durante a graduação.

$\mathrm{O}$ fato de não ter ocorrido associação entre as variáveis independentes e escore dos grupos sugere que o conhecimento adquirido sobre transtornos mentais no período pós-clínico é o grande marco para uma atitude positiva em relação ao suicídio.No entanto, com relação ao aprendizado, a maioria das faculdades de Medicina não incentiva o ensino de Psiquiatria ou o faz de forma precária. Como resultado, os alunos não armazenam as informações necessárias e nem desenvolvem habilidades para o diagnóstico e tratamento dos transtornos psiquiátricos prevalentes, o que é crítico para aqueles com risco de suicídio.

A literatura aponta que estudantes de Medicina e de Psicologia, bem como os médicos de forma geral, recebem pouco treinamento sobre prevenção ao suicídio e que as atitudes negativas em relação ao suicídio são comuns entre os estudantes de Medicina, embora algumas dessas atitudes possam mudar ao longo dos anos de formação médica ${ }^{11,12}$.Um treinamento sobre suicídio realizado em Hong Kong evidenciou atitudes positivas em relação ao suicídio após a participação em módulo específico sobre o tema. Ao final do curso, verificou-se redução da avaliação negativa, diminuição do estigma e aumento da sensibilidade aos fatos relacionados com o suicídio $^{12}$. 
A comparação de estudos realizados em diferentes países mostra que os fatores culturais também desempenham um importante papel nas atitudes em relação ao suicídio. Geralmente, em países desenvolvidos, as atitudes tendem a ser mais permissivas, sendo mais restritivas nos países em desenvolvimento $^{13}$. Em regiões onde as tentativas de suicídio são elevadas, as atitudes tendem a ser mais hostis e intolerantes em relação ao comportamento suicida em comparação com regiões que exibem taxas mais baixas.

Um estudo que comparou estudantes americanos e japoneses não revelou diferenças culturais em suas atitudes para com o suicídio, mas observou diferenças relacionadas a gênero e crença religiosa ${ }^{14}$. Outro trabalho, que avaliou estudantes indianos e austríacos, mostrou atitudes mais restritivas entre os asiáticos, que rejeitaram o direito de cometer suicídio e o consideraram um ato de covardia ou associado a um transtorno mental. Por outro lado, estudantes vienenses apresentaram atitudes mais permissivas, minimizando a influência das doenças mentais no comportamento suicida ${ }^{15}$.

As atitudes dos profissionais que lidam com pacientes suicidas têm sido examinadas em diversos estudos. Entrevistas e questionários dirigidos a médicos e enfermeiros têm revelado atitudes negativas em relação àqueles que tentam suicídio, especialmente nos casos em que o profissional percebe pequena intenção suicida no paciente. Em relação a diferentes setores de atendimento, os profissionais que trabalham em emergência apresentam mais atitudes negativas quando comparados aos que trabalham em unidades de cuidados intensivos, que exibem mais atitudes positivas em relação a esses pacientes ${ }^{16}$.

Avaliar um paciente suicida comumente desperta fortes sentimentos no médico examinador, especialmente ansiedade por um erro de conduta e uma consequência catastrófica. $\mathrm{O}$ profissional pode experimentar também sentimentos de impotência e mobilizar emoções de caráter negativo Quando a tentativa de suicídio envolve um estudante de Medicina, a equipe de atendimento geralmente o hostiliza. $\mathrm{O}$ aluno passa a ser considerado um fraco. Médicos que trataram de pacientes suicidas sabem que, frequentemente, a reação imediata daqueles que cercam o paciente é serem cruéis e rejeitá-1o ${ }^{17}$.

Os profissionais de saúde, em especial os que atuam em setores de emergência, lidam constantemente com indivíduos em situação de crise e que tentam suicídio. De forma semelhante, aqueles que atuam na Atenção Básica, por estarem em contato próximo e prolongado com os pacientes, seus familiares e sua comunidade, estão em posição privilegiada para avaliação dos pacientes em risco de suicídio.
No entanto, esses profissionais, especialmente os médicos, não estão preparados de forma adequada para atender pacientes suicidas, o que pode gerar opiniões desfavoráveis, reações negativas e intolerância, prejudicando o processo terapêutico. As dificuldades dos profissionais em lidar com os diferentes aspectos relacionados à morte, e em especial ao suicídio, podem desencadear conflitos emocionais naqueles que lidam com esses pacientes ${ }^{18}$.

Esses conflitos podem significar dificuldades na abordagem do tema dentro da equipe de saúde e no relacionamento com o próprio paciente ou com seus familiares. Essa postura pode interferir na atuação profissional, diminuindo sua eficácia, ou, então, associar-se à incapacidade para controlar o sofrimento do paciente.

Há oito anos, o Ministério da Saúde elaborou um Plano Nacional de Prevenção do Suicídio ${ }^{19,20}$, e entre os principais objetivos a alcançar se destacam a promoção e a educação permanente dos profissionais de saúde da Atenção Básica, dos serviços de saúde mental, das unidades de urgência e emergência, de acordo com os princípios da integralidade e da humanização.

No entanto, pouco se discute sobre as atitudes dos estudantes e dos profissionais frente ao suicídio, as quais são um importante componente dessas estratégias, inclusive do ponto de vista terapêutico. O conhecimento e a discussão do tema pelos alunos, junto a atividades e estágios curriculares, contribuem favoravelmente para a incorporação de atitudes positivas frente à morte e ao suicídio.

\section{CONCLUSÃO}

No âmbito educacional, as atitudes dos estudantes em relação à morte e ao suicídio adquirem relevância especial, dada a existência de uma relação definida entre atitudes e aprendizagem. Essas atitudes compõem parte importante das habilidades afetivas que podem ser ensinadas e apreendidas pelos alunos. A incorporação dessas atitudes em relação aos diferentes aspectos envolvidos na atenção à saúde constitui um poderoso preditor dos comportamentos e ações dos estudantes (e dos futuros profissionais) em relação aos pacientes.

As atitudes dos estudantes e dos profissionais no atendimento são importantes porque seu conhecimento e sua visão sobre o suicídio, bem como a real disposição em ajudar esses pacientes podem influenciar de forma significativa a continuidade do tratamento. E desde que as atitudes dos estudantes e dos profissionais em particular, e da população em geral, podem revelar um comportamento futuro, elas são fundamentais na prevenção e tratamento de indivíduos que tentam suicídio. 


\section{REFERÊNCIAS}

1. WHO. Multisite intervention study on suicidal behaviors. Geneva, 2002 [capturado em: 20 set. 2013]. Disponível em: http://www.who.int/mental_health/56.pdf.

2. Minayo MCS. Suicídio: violência auto-infligida. In: Secretaria de Vigilância em Saúde. Impacto da violência na saúde dos brasileiros. Brasília: MS; 2005. p. 205-39.

3. Bertolote JM, Fleischmann A. A global perspective in the epidemiology of suicide. Suicidology. 2002;7(2):6-8.

4. Lake CR. How Academic Psychiatry can better prepare students for their future patients. Part I: The failure to recognize depression and risk for suicide in primary care; problem identification, responsibility, and solutions. Behavl Med.2008;34:95-9.

5. Schulberg HC, Lee PW, Bruce ML. Suicidal ideation and risk levels among primary-care patients with uncomplicated depression. Ann Fam Med. 2005;(3):523-8.

6. Nutting PA, Dickinson LM, Rubenstein LV, Keeley RD, Smith JL, Elliott CE. Improving detection of suicidal ideation among depressed patients in primary care. Ann Fam Med. 2005;(3):529-36.

7. Gaynes BN, West SL, Ford CA, Frame P, Klein J, Lohr KN. Screening for suicide risk in adults: a summary of the evidence for the US Preventive Services Task Force. Ann Intern Med. 2004;140:822-35.

8. Botega NJ, Reginato DG, Silva SV, Cais CFS; Rapeli CB; Mauro MLF et al.Nursing personnel attitudes towards suicide: the development of a measure scale. Rev Bras Psiq, 2005; 27(4):315-18. Acesso em: 11 fev. 2009.

9. Troncon LEA, Colares MFA, Figueiredo JFC, Cianflone ARL, Rodrigues MLV, Piccinato CE, et al. Atitudes de graduandos em medicina em relação a aspectos relevantes da prática médica. Rev Bras Educ Med. 2003;27(1):10-8.

10. Mascia AR, Silva FB, Lucchese AC, De Marco MA, Martins MCF, Martins LAN. Atitudes frente a aspectos relevantes da prática médica: estudo transversal randomizado com alunos de segundo e sexto anos. Rev Bras Educ Med. 2009;33(1):40-8.

11. Emul M, Uzunoglu Z, Sevinç H, Güzel C, Yilmaz C, Erkut $\mathrm{D}$, et al. The attitudes of preclinical and clinical turkish medical students toward suicide attempters. Crisis. 2011;32(3):128-33.

12. Yousuf S, Beh PSL, Wong PWC. Attitudes towards suicide following an undergraduate suicide prevention module: experience of medical students in Hong Kong. Hong Kong Med J. 2013;19:377-85.
13. Hjelmeland H, Akotia CS, Owens V, Knizek BL, Nordvik $\mathrm{H}$, Schroeder R, et al. Self-reported suicidal behavior and attitudes toward suicide and suicide prevention among psychology students in Ghana, Uganda, and Norway. Crisis.2008;29(1):20-31

14. Domino G, Takashi Y. Attitudes toward suicide in japanese and american medical students. Suicide Life Threat Behav.1991;21:345-58.

15. Etzersdorfer E, Vijayakumar L, Schony W, Grausgruber A, Sonneck G. Attitudes towards suicide among medical students: comparison between Madras (India) and Vienna (Austria). Soc PsychiatPsychiatEpidemiol. 1998;33:104-10.

16. McAllister M, Creedy D, Moyle W, Farrugia C. Nurses' attitudes towards clients who self-harm. J Adv Nurs. 2002;40(5):578-86.

17. Meleiro AMAS. Suicídio entre médicos e estudantes de medicina. Rev Ass Med Bras.1998;44(2):135-40.

18. Öncü B, Soykan C, Shan SO, Sayil I. Attitudes of medical students, general practitioners, teachers, and police officers toward suicide in a turkish sample. Crisis. 2008;29(4):173-9.

19. Ministério da Saúde. Estratégia nacional de prevenção do suicídio: manual dirigido a profissionais das equipes de saúde mental. Brasília; 2006. [capturado em: 30 out. 2012]. Disponível em: www.saude.gov.br

20. Ramos INB, Falcão EBM. Suicídio: um tema pouco conhecido na formação médica. Rev. bras. educ. med.2011;35(4) . [capturado em 22 dez. 2013]; 507-16. Disponível emhttp:/ / www.scielo.br

\section{CONTRIBUIÇÃO DOS AUTORES}

Carlos Eduardo Leal Vidal contribuiu na concepção, desenho, análise e interpretação dos dados, elaboração do artigo e na aprovação da versão final. Todos os demais autores contribuíram significativamente na concepção, no desenho, coleta e interpretação dos dados, na elaboração do artigo e na aprovação da versão final a ser publicada.

\section{CONFLITO DE INTERESSES}

Declarou não haver.

\section{ENDEREÇO PARA CORRESPONDÊNCIA}

Claudia Antunes Magalhães

Rua Professor Orozimbo Melo, 122

Bairro Retiro - Lavras

CEP 37200-000- MG

E-mail: claudia.magalhaes@yahoo.com.br 